CHIBA-EP-71

October 1993

\title{
The Stochastic Quantization Method in Phase Space and a New Gauge Fixing Prodedure
}

\author{
Riuji Mochizuki* \\ Department of Physics, Chiba University, \\ 1-33 Yayoi-cho, Inage-ku, Chiba 263, Japan
}

\section{Abstract}

We study the stochastic quantization of the system with first class constraints in phase space. Though the Langevin equations of the canonical variables are defined without ordinary gauge fixing procedure, gauge fixing conditions are automatically selected and introduced by imposing stochastic consistency conditions upon the first class constraints. Then the equilibrium solution of the Fokker-Planck equation is identical with corresponding path integral distribution.

\footnotetext{
* e-mail address: mochi@cuphd.nd.chiba-u.ac.jp
} 


\section{2. $§ 1$. INTRODUCTION}

The stochastic quantization method, which has been proposed by Parisi and $\mathrm{Wu}^{[1]}$ in 1981 , is known as the third quantization method ${ }^{[2]}$ following the canonical and path integral formalism. In the scheme fields $\phi(x)$ have an additional coordinate $t$;

$$
\phi(x) \rightarrow \phi(x, t),
$$

coordinate which is called the fictitious time. The development of the fields with the lapse of the fictitious time is described by a Langevin equation

$$
\begin{aligned}
d \phi(x, t) & \equiv \phi(x, t+d t)-\phi(x, t) \\
& =-\left.\frac{\partial \mathrm{L}}{\partial \phi}\right|_{\phi=\phi(x, t)}+d W(x, t),
\end{aligned}
$$

where $\mathrm{L}$ is the Lagrangian of a system and $d W$ is the Wiener process whose correlations are defined as

$$
\begin{gathered}
\langle d W(x, t)\rangle=0 \\
\left\langle d W(x, t) d W\left(x^{\prime}, t\right)\right\rangle=2 \delta\left(x-x^{\prime}\right) d t .
\end{gathered}
$$

The equations enable us to calculate correlation functions of the fields, which tend to corresponding Green functions in the equilibrium limit $t \rightarrow \infty$.

The correlation functions may be given in another form

$$
\langle\cdots\rangle=\int D \phi \cdots P(\phi, t),
$$

where $P$ is a probability distribution. A Fokker-Planck equation, which $P$ satisfies, is obtained by calculating, with the Langevin equation, the correlation function of the fictitious time derivative of any functional $f(\phi)$ and comparing it with 
$\int D \phi f \cdot \partial P / \partial t$. The Fokker-Planck equation thus becomes

$$
\frac{\partial P}{\partial t}=\frac{\partial}{\partial \phi}\left\{\frac{\partial \mathrm{L}}{\partial \phi}+\frac{\partial}{\partial \phi}\right\} P
$$

The equation has in the equilibrium limit $\partial P / \partial t=0$ a solution

$$
P=\exp (-\mathrm{L})
$$

which is the same as corresponding path integral distribution.

It is one of the large merits of the stochastic quantization method that it enables us to quantize gauge fields without gauge fixing conditions. ${ }^{[3]}$ Hence if we extend the stochastic quantization method, which is ordinary defined in configuration space, to phase space, we may shed light on the quantization of the systems with first class constraints (FCC). Until now Namiki et al. ${ }^{[4]}$ and $\mathrm{we}^{[5]}$ have studied how to handle constraints by the stochastic quantization method in configuration space (the SQM-cs), showing that it is much useful to impose the consistency condition with the lapse of the fictitious time, the stochastic consistency condition, upon the constraints. On the other hand, some proposals for the stochastic quantization method in phase space (the SQM-ps) have been made. ${ }^{[6]}$ Ohba ${ }^{[7]}$ has tried to quantize by the SQM-ps, in particular, the system with FCC where gauge fixing conditions are added. It is accordingly the system with second class constraints that he quantized by the SQM-ps.

In this paper we quantize the system with FCC by the SQM-ps without usual gauge fixing procedure and show that gauge fixing conditions are automatically introduced through the stochastic consistency conditions upon the FCC. Since the prescription to impose them is straightforward and has little dependence on the form of the FCC, it will be useful when we quantize complicatedly constrained fields and may bring us the other knowledge about gauge theory than the other quantization methods have offered.

This paper is organized as follows. In $\S 2$, taking notice of the relation between the SQM-cs and the SQM-ps and, in particular, the Lee-Yang term, we define the strict form of the Langevin equations with a constant kernel, of canonical variables. 
In $\S 3$, we show how to apply the SQM-ps to the system with FCC. We impose the stochastic consistency conditions upon the FCC and the determinant factor which appears with the gauge fixing procedure consequently becomes included in the solution of the Fokker-Planck equation in the equilibrium limit. In $\S 4$, we define new fields and divide them into constraint variables and independent ones to clarify what gauge the stochastic consistency conditions accord to. $\S 5$ is devoted to the conclusion. Besides them, we give in the appendix how to apply our formulation to the system with second class constraints (FCC and gauge fixing conditions).

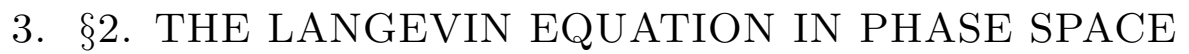

In this section we consider the system with the Lagrangian in Euclidean spacetime

$$
\begin{gathered}
\mathrm{L}=\frac{1}{2} G_{A B}(q) \partial_{\mu} q^{A} \partial_{\mu} q^{B}, \\
A, B=1, \cdots, N \\
\mu=0, \cdots, n .
\end{gathered}
$$

For such a system as includes a field-dependent metric, by using Ito's calculation rule, ${ }^{[8]}$ the Langevin equation in configuration space is

$$
d q^{A}(t)=-G^{A B} \frac{\partial \mathrm{L}}{\partial q^{B}} d t+\frac{1}{\sqrt{G}} \frac{\partial}{\partial q^{B}}\left(\sqrt{G} G^{A B}\right) d t+E_{a}^{A} d W^{a},
$$

where $G^{A B}$ is the inverse of the metric $G_{A B}$ and $E_{a}^{A}$ is the vielbein defined with its inverse $E_{A}^{a}$ as

$$
\begin{gathered}
E_{a}^{A} E_{b}^{B} \delta^{a b}=G^{A B}, \\
E_{A}^{a} E_{B}^{b} \delta_{a b}=G_{A B}, \\
E_{a}^{A} E_{B}^{a}=\delta_{B}^{A}, \quad E_{a}^{A} E_{A}^{b}=\delta_{a}^{b}, \\
a, b=1, \cdots, N . \text { : reference frame indices }
\end{gathered}
$$

The additional term of the Langevin equation (2.2) is necessary for general coordinate transformation (GCT) covariance $^{[9]}$ and, in perturbation, cancellation of 
divergent terms. ${ }^{[5]}$ Moreover we can observe it induces the Lee-Yang term ${ }^{[10]}$ in equilibrium Fokker-Planck distribution. ${ }^{[11]}$ The Fokker-Planck equation in this case is

$$
\begin{gathered}
\frac{\partial P}{\partial t}=\frac{\partial}{\partial q^{A}} G^{A B}\left\{\frac{\partial \mathrm{L}}{\partial q^{B}}-\frac{1}{\sqrt{G}} \frac{\partial \sqrt{G}}{\partial q^{B}}+\frac{\partial}{\partial q^{B}}\right\} P, \\
G \equiv \operatorname{det}\left(G_{A B}\right),
\end{gathered}
$$

the equilibrium solution of which is

$$
P=\exp \left(-\mathrm{L}+\frac{1}{2} \ln G\right)
$$

The correlation function is thereby written as

$$
\langle\cdots\rangle=\int D q \cdots \exp \left(-\mathrm{L}+\frac{1}{2} \ln G\right)
$$

which is consistent with the path integral formalism.

Let us shift to phase space. First we define the conjugate momentum $p_{A}$ of $q^{A}$ and the Hamiltonian $H$ as

$$
\begin{gathered}
p_{A}=+i \frac{\partial \mathrm{L}}{\partial\left(\partial_{0} q^{A}\right)}=i G_{A B} \partial_{0} q^{B} \\
H=+i p_{A} \partial_{0} q^{A}+\mathrm{L}=\frac{1}{2} G^{A B} p_{A} p_{B}+\frac{1}{2} G_{A B} \partial_{i} q^{A} \partial_{i} q^{B} .
\end{gathered}
$$

The equations of motion in phase space are the Hamilton equations

$$
\begin{aligned}
& -i \partial_{0} p_{A}-\frac{\partial H}{\partial q^{A}}=0, \\
& +i \partial_{0} q^{A}-\frac{\partial H}{\partial p_{A}}=0,
\end{aligned}
$$

in place of the Euler-Lagrange equation in configuration space. It is natural to adopt the LHS of the Hamilton equations (2.5) as naive drift terms of the Langevin 
equations in the phase space. Moreover since the metric of the manifold spanned by the canonical variables $q$ and $p$ is

$$
\left(\begin{array}{rl}
G_{A B}(q) & 0 \\
0 & G^{A B}(q)
\end{array}\right)
$$

determinant of which is unity, the GCT covariant Langevin equations are defined in our formulation as

$$
\left\{\begin{array}{l}
d q^{A}=G^{A B}\left(-i \partial_{0} p_{B}-\frac{\partial H}{\partial q^{B}}\right) d t+\frac{\partial G^{A B}}{\partial q^{B}} d t+E_{a}^{A} d W^{a} \\
d p_{A}=C G_{A B}\left(+i \partial_{0} q^{B}-\frac{\partial H}{\partial p_{B}}\right) d t+\sqrt{C} E_{A}^{a} d V_{a}
\end{array}\right.
$$

where $C$ is the constant kernel determined later and $d W^{a}$ and $d V_{a}$ are the Wiener process defined to have following correlations:

$$
\begin{gathered}
\left\langle d W^{a}(x, t) d W^{b}\left(x^{\prime}, t\right)\right\rangle=2 \delta^{a b} \delta^{n}\left(x-x^{\prime}\right) d t \\
\left\langle d V_{a}(x, t) d V_{b}\left(x^{\prime}, t\right)\right\rangle=2 \delta_{a b} \delta^{n}\left(x-x^{\prime}\right) d t \\
\left\langle d W^{a}\right\rangle=\left\langle d V_{a}\right\rangle=\left\langle d W^{a} d V_{b}\right\rangle=0 .
\end{gathered}
$$

The probability distribution $P(q, p, t)$ in the phase space is also defined through correlation functions as

$$
\langle\cdots\rangle=\int D q D p \cdots P(q, p, t)
$$

Similar calculation to the case in the configuration space leads the Fokker-Planck equation

$$
\frac{\partial P}{\partial t}=\left[\frac{\partial}{\partial q^{A}} G^{A B}\left\{\frac{\partial L}{\partial q^{B}}+\frac{\partial}{\partial q^{B}}\right\}+C G_{A B} \frac{\partial}{\partial p_{A}}\left\{\frac{\partial L}{\partial p_{B}}+\frac{\partial}{\partial p_{B}}\right\}\right] P
$$

where $L$ is the Lagrangian in the phase space defined as

$$
L \equiv-i p_{A} \partial_{0} q^{A}+H
$$

The Fokker-Planck equation has a solution in the equilibrium limit with which the 
correlation function is rewritten as

$$
\langle\cdots\rangle=\int D q D p \cdots \exp (-L) .
$$

The constant kernel $C$, introduced for dimensional consistency between the Langevin equations (2.6), has the same dimension as $t^{-1}$. It is fixed if we demand that the Langevin equations in the phase space (2.6) are reduced to the Langevin equation in the configuration space (2.2). For the purpose, we put the Hamiltonian (2.4) into Eqs.(2.6):

$$
\left\{\begin{aligned}
d q^{A}= & {\left[-i G^{A B} \partial_{0} p_{B}-\frac{G^{A B}}{2}\left(\frac{\partial G^{C D}}{\partial q^{B}} p_{C} p_{D}+\frac{\partial G_{C D}}{\partial q^{B}} \partial_{i} q^{C} \partial_{i} q^{D}\right)\right.} \\
& \left.+G^{A B} \partial_{i}\left(G_{B C} \partial_{i} q^{C}\right)+\frac{\partial G^{A B}}{\partial q^{B}}\right] d t+E_{a}^{A} d W^{a} \\
d p_{A}= & C\left[i G_{A B} \partial_{0} q^{B}-p_{A}\right] d t+\sqrt{C} E_{A}^{a} d V_{a}
\end{aligned}\right.
$$

We eliminate the momenta in Eqs.(2.8) and obtain the Langevin equation of $q^{A}$

$$
\begin{aligned}
d q^{A}=[ & -i G^{A B} \partial_{0}\left(i G_{B C} \partial_{0} q^{C}+\frac{E_{B}^{a} d V_{a}}{\sqrt{C} d t}-\frac{d p_{B}}{C d t}\right)-\frac{G^{A B}}{2}\left\{\frac{\partial G_{C D}}{\partial q^{B}} \partial_{i} q^{C} \partial_{i} q^{D}\right. \\
& \left.+\frac{\partial G^{C D}}{\partial q^{B}}\left(i G_{C E} \partial_{0} q^{E}+\frac{E_{C}^{a} d V_{a}}{\sqrt{C} d t}-\frac{d p_{C}}{C d t}\right)\left(i G_{D F} \partial_{0} q^{F}+\frac{E_{D}^{b} d V_{b}}{\sqrt{C} d t}-\frac{d p_{D}}{C d t}\right)\right\} \\
& \left.+G^{A B} \partial_{i}\left(G_{B C} \partial_{i} q^{C}\right)+\frac{\partial G^{A B}}{\partial q^{B}}\right] d t+E_{a}^{A} d W^{a}
\end{aligned}
$$

Taking the correlation of the Wiener process $d V_{a}$ in the limit $d t \rightarrow 0$, Eq.(2.9) should be identical with (2.2). It is realized if

$$
C=\frac{2}{d t}
$$

which is also supported in view of the dimension. We therefore conclude that the Langevin equations of the canonical variables $q^{A}$ and $p_{A}$ are

$$
\left\{\begin{array}{l}
d q^{A}=-G^{A B} \frac{\partial L}{\partial q^{B}} d t+\frac{\partial G^{A B}}{\partial q^{B}} d t+E_{a}^{A} d W^{a} \\
d p_{A}=-2 G_{A B} \frac{\partial L}{\partial p_{B}}+\sqrt{\frac{2}{d t}} E_{A}^{a} d V_{a}
\end{array}\right.
$$

The drift term (the first term on the RHS) of the latter equation of (2.11) being 
very powerful because of the order of $d t, p_{A}$ will reaches an equilibrium state in a very short time. It justifies the manipulation to connect Eq.(2.2) with Eq.(2.9); taking the correlation only of $d V_{a}$ accords to thinking the system at a finite time. The discussion on the constant kernel in this section may also be applied when we write the Langevin equation of an auxiliary field, the kinetic term of which does not exist in the Lagrangian.

\section{4. §3. FIRST CLASS CONSTRAINTS AND STOCHASTIC CONSIS- TENCY CONDITIONS}

In this section we consider the system, the Lagrangian of which is $\mathrm{L}\left(q^{I}, \partial_{0} q^{I}\right)$ $(I=1, \cdots, N)$, with FCC

$$
F^{a}(q, p)=0, \quad(a=1, \cdots, M)
$$

The conjugate momentum $p_{I}$ and the Hamiltonian $H$ are defined through Eqs. (2.3) and (2.4) respectively and the metric of the system is assumed to be $\delta_{I J}$ for simplicity. Following the canonical approach the Hamiltonian is generalized to include the FCC as

$$
H_{G} \equiv H+\lambda_{a} F^{a},
$$

where $\lambda_{a}$ is a multiplier field, which cannot be determined without gauge fixing in the canonical formalism. We define the Langevin equations accordingly as

$$
\begin{aligned}
& d q^{I}=-\frac{\partial L_{G}}{\partial q^{I}} d t+d W^{I}, \\
& d p^{I}=-C \frac{\partial L_{G}}{\partial p^{I}} d t+\sqrt{C} d V^{I},
\end{aligned}
$$

where the singular Lagrangian $L_{G}$ is

$$
L_{G} \equiv-i p^{I} \partial_{0} q^{I}+H_{G}
$$

It is convenient to define symplectic variables

$$
\phi^{i} \equiv\left\{\frac{p^{1}}{\sqrt{C}}, \cdots, \frac{p^{N}}{\sqrt{C}}, q^{1}, \cdots, q^{N}\right\}
$$




$$
d Y^{i} \equiv\left\{d V^{1}, \cdots, d V^{N}, d W^{1}, \cdots, d W^{N}\right\}
$$

with which we can rewrite the Langevin equation in a simple form

$$
d \phi^{i}=-\frac{\partial L_{G}}{\partial \phi^{i}} d t+d Y^{i}
$$

When we quantize gauge field with the Langevin equation in configuration space, we do not need to fix the gauge freedom; the Faddeev-Popov ghost effects are automatically carried into correlation functions and divergence, though appearing in the correlation functions, does not in physical quantities. ${ }^{[3]}$ The Langevin equation (3.4), however, is not suitable for perturbation because of the multipliers, and neither is the Fokker-Planck formulation. The Fokker-Planck equation derived through the Langevin equation (3.4) is

$$
\frac{\partial P}{\partial t}=\frac{\partial}{\partial \phi^{i}}\left\{\frac{\partial L_{G}}{\partial \phi^{i}}+\frac{\partial}{\partial \phi^{i}}\right\} P
$$

whose solution in the equilibrium limit is

$$
P=\exp \left(-L_{G}\right)
$$

We cannot obtain proper correlation functions either by using the distribution (3.6).

We need to change the Langevin equation (3.4) into the form which fits for our handling. We, following the case in the configuration space, ${ }^{[4[5]}$ try to impose on each FCC (3.1) the stochastic consistency condition

$$
d F^{a}=0
$$

and hence determine the multiplier $\lambda_{a}$. Our strategy may seem strange since the multipliers for FCC cannot be fixed in the canonical formalism. We will however accomplish the purpose as the following. If we take Ito's formula ${ }^{[8]}$ into consideration, the stochastic consistency condition (3.7) becomes

$$
0=d F^{a}
$$




$$
\begin{aligned}
= & \frac{\partial F^{a}}{\partial \phi^{i}} d \phi^{i}+\frac{1}{2} \frac{\partial^{2} F^{a}}{\partial \phi^{i} \partial \phi^{j}}\left\langle d \phi^{i} d \phi^{j}\right\rangle_{O(d t)} \\
= & \frac{\partial F^{a}}{\partial \phi^{i}}\left\{\left(-\frac{\partial L}{\partial \phi^{i}}-\lambda_{b} \frac{\partial F^{b}}{\partial \phi^{i}}\right) d t+d Y^{i}\right\} \\
& +\frac{1}{2} \frac{\partial^{2} F^{a}}{\partial \phi^{i} \partial \phi^{j}}\left\langle d \phi^{i} d \phi^{j}\right\rangle_{O(d t)},
\end{aligned}
$$

where $\left\langle d \phi^{i} d \phi^{j}\right\rangle_{O(d t)}$ is the correlation whose order is $d t$. It is not $2 \delta^{i j} d t$ since $\lambda_{a}$ contains the Wiener process $d Y^{i}$ and hence after setting the multipliers concretely we will compute it. ${ }^{[5112]}$ We change Eq. (3.8) into

$$
D^{a b} \lambda_{b}=-\frac{\partial F^{a}}{\partial \phi^{i}} \frac{\partial L}{\partial \phi^{i}}+\frac{1}{2 d t} \frac{\partial^{2} F^{a}}{\partial \phi^{i} \partial \phi^{j}}\left\langle d \phi^{i} d \phi^{j}\right\rangle_{O(d t)}+\frac{\partial F^{a}}{\partial \phi^{i}} \frac{d Y^{i}}{d t},
$$

where

$$
D^{a b} \equiv \frac{\partial F^{a}}{\partial \phi^{i}} \frac{\partial F^{b}}{\partial \phi^{i}}
$$

Assuming that $D^{a b}$ has the inverse $D_{a b}^{-1}$, we obtain the definite form of $\lambda_{a}$

$$
\lambda_{a}=D_{a b}^{-1}\left\{-\frac{\partial F^{b}}{\partial \phi^{i}} \frac{\partial L}{\partial \phi^{i}}+\frac{1}{2 d t} \frac{\partial^{2} F^{b}}{\partial \phi^{i} \partial \phi^{j}}\left\langle d \phi^{i} d \phi^{j}\right\rangle_{O(d t)}+\frac{\partial F^{b}}{\partial \phi^{i}} \frac{d Y^{i}}{d t}\right\} .
$$

The Langevin equaiton (3.4), into which Eq.(3.11) is put, becomes

$$
d \phi^{i}=K^{i j}\left\{-\frac{\partial L}{\partial \phi^{j}} d t+d Y^{j}\right\}-\frac{1}{2} \frac{\partial F^{a}}{\partial \phi^{i}} D_{a b}^{-1} \frac{\partial^{2} F^{b}}{\partial \phi^{j} \partial \phi^{k}}\left\langle d \phi^{j} d \phi^{k}\right\rangle_{O(d t)},
$$

where $K^{i j}$ is the projection operator which extracts the quotient space composed of only the independent variables:

$$
\begin{gathered}
K^{i j} \equiv \delta^{i j}-R^{i j}, \\
R^{i j} \equiv \frac{\partial F^{a}}{\partial \phi^{i}} D_{a b}^{-1} \frac{\partial F^{b}}{\partial \phi^{j}} .
\end{gathered}
$$

In this stage we can compute $\left\langle d \phi^{i} d \phi^{j}\right\rangle_{O(d t)}$ by using the Langevin equation (3.12) 
and finally obtain the Langevin equation without the multipliers:

$$
d \phi^{i}=K^{i j}\left\{-\frac{\partial L}{\partial \phi^{j}} d t+d Y^{j}\right\}-\frac{\partial F^{a}}{\partial \phi^{i}} D_{a b}^{-1} \frac{\partial^{2} F^{b}}{\partial \phi^{j} \partial \phi^{k}} K^{j k} d t
$$

which is the desirable form for calculation and of course satisfies Eq. (3.7). Imposing the stochastic consistency condition thereby enables us to determine the multipliers for the FCC, which fact suggests that the stochastic consistency condition may be equivalent to some gauge fixing condition.

The Fokker-Planck equation, expected to bring us more information, is

$$
\frac{\partial P(\phi, t)}{\partial t}=\frac{\partial}{\partial \phi^{i}} K^{i j}\left\{\frac{\partial L}{\partial \phi^{j}}-\frac{\partial^{2} F^{a}}{\partial \phi^{j} \partial \phi^{k}} D_{a b}^{-1} \frac{\partial F^{b}}{\partial \phi^{k}}+\frac{\partial}{\partial \phi^{j}}\right\} P(\phi, t)
$$

Taking the FCC (3.1) into consideration, we write the equilibrium solution as

$$
\begin{gathered}
P=\int D \lambda \sqrt{D} \exp \left(-L-\lambda_{a} F^{a}\right) \\
D \equiv \operatorname{det}\left(D^{a b}\right)
\end{gathered}
$$

where appears the determinant factor, which does not exist in the previous solution (3.6). We hence expect that the FCC (3.1) and the gauge fixing conditions, which we will write as $C^{a}=0$, due to the stochastic consistency conditions (3.7), constitute the second class constraints whose Poisson brackets satisfy

$$
\operatorname{det}\left\{F^{a}, C^{b}\right\}_{P . B .}=\sqrt{D}
$$

The probability distribution (3.15), however, seems to include no gauge fixing term and diverge in the same manner as the previous one (3.6). Is the guess wrong ? If not, we must find the finite solution of the Fokker-Planck equation (3.14). The answer will be obtained in the next section. 


\section{5. §4. THE STOCHASTIC CONSISTENCY CONDITION AS GAUGE FIXING}

Our purpose in this section is to confirm the above mentioned conjecture by investigating in more detail the Langevin equation (3.13) and the Fokker-Planck equation (3.14), for which reason it is necessary to introduce new symplectic variables and divide them into constraint and independent variables. We write the new variables as

$$
\Phi^{\mu} \equiv\left\{\frac{\Pi^{1}}{\sqrt{C}}, \cdots, \frac{\Pi^{N}}{\sqrt{C}}, Q^{1}, \cdots, Q^{N}\right\}, \quad(\mu=1, \cdots, 2 N)
$$

where $Q$ and $\Pi$ are canonical conjugate of each other. Vielbein fields are, connecting the new variables with the old ones, defined as

$$
\begin{gathered}
E_{i}^{\mu} \equiv \frac{\partial \Phi^{\mu}}{\partial \phi^{i}}, \\
E_{i}^{\mu} E_{\nu, i}=\delta_{\nu}^{\mu}, \quad E_{i}^{\mu} E_{\mu, j}=\delta_{i j} .
\end{gathered}
$$

Besides the definition, since $Q$ and $\Pi$ are the canonical variables, the vielbein fields must satisfy the following relations:

$$
\left\{\begin{aligned}
E^{\alpha+N}{ }_{I+N} & =E_{\alpha, I}, \\
E_{I}^{\alpha+N} & =-E_{\alpha, I+N}, \\
E_{\alpha+N, I+N} & =E^{\alpha}{ }_{I}, \\
E_{\alpha+N, I} & =-E^{\alpha}{ }_{I+N} .
\end{aligned} \quad(\alpha=1, \cdots, N ; I=1, \cdots, N)\right.
$$

Each of the constraints $F^{a}$, assumed to be the first class, may be transfered ${ }^{[13]}$ to the new momentum $\Pi^{a} / \sqrt{C}$, that is, we set the vielbein fields (4.2) with the left-hand indices $\mu=a(a=1, \cdots, M)$ as

$$
E_{i}^{a}=\frac{\partial F^{a}}{\partial \phi^{i}}, \quad E_{a, i}=D_{a b}^{-1} \frac{\partial F^{b}}{\partial \phi^{i}} .
$$


From Eqs. (4.4) and (4.5) we set those with $\mu=a+N$ :

$$
\left\{\begin{aligned}
E_{I}^{a+N} & =-D_{a b}^{-1} \frac{\partial F^{b}}{\partial \phi^{I+N}} \\
E_{I+N}^{a+N} & =D_{a b}^{-1} \frac{\partial F^{b}}{\partial \phi^{I}} \\
E_{a+N, I} & =-\frac{\partial F^{a}}{\partial \phi^{I+N}} \\
E_{a+N, I+N} & =\frac{\partial F^{a}}{\partial \phi^{I}}
\end{aligned}\right.
$$

There are some more relations which Eqs. (4.4) and (4.5) yield:

$$
\left\{\begin{aligned}
E_{i}^{a} E_{i}^{b} & =E_{a+N, i} E_{b+N, i}=D^{a b} \\
E_{a, i} E_{b, i} & =E_{i}^{a+N} E_{i}^{b+N}=D_{a b}^{-1}, \\
E_{i}^{a} E_{i}^{b+N} & =E_{a, i} E_{i}^{b+N}=E_{i}^{a} E_{b+N, i}=E_{a, i} E_{b+N, i}=0, \\
R_{i j} & =E_{i}^{a} E_{a, j}, \\
K_{i j} & =E^{a+N} E_{a+N, j}+E_{i}^{A} E_{A, j}+E_{i}^{A+N} E_{A+N, j}, \\
( & A=M+1, \cdots, N) .
\end{aligned}\right.
$$

The third equation of (4.7) is obtained with the help of the assumption that the constraints (3.1) are the first class. Moreover $E_{i}^{A}$ and $E_{i}^{A+N}$ chosen orthogonal to each other and to the other vielbein fields, the metric of the manifold spanned by the independent variables $\Phi^{A}, \Phi^{a+N}$ and $\Phi^{A+N}$ is

$$
G_{\mu^{\prime} \nu^{\prime}}=\left(\begin{array}{rcl}
g_{A B} & 0 & 0 \\
0 & D^{a b} & 0 \\
0 & 0 & g^{A B}
\end{array}\right), \quad\left(\mu^{\prime} \nu^{\prime}=M+1, \cdots, 2 N\right)
$$

with

$$
\begin{aligned}
& g^{A B}=E_{i}^{A} E_{i}^{B}=E_{A+N, i} E_{B+N, i}, \\
& g_{A B}=E_{A, i} E_{B, i}=E_{i}^{A+N} E_{i}^{B+N} .
\end{aligned}
$$

We thus obtain the Langevin equations of the new independent variables:

$$
d \Phi^{\mu^{\prime}}=\frac{\partial \Phi^{\mu^{\prime}}}{\partial \phi^{i}} d \phi^{i}+\frac{\partial^{2} \Phi^{\mu^{\prime}}}{\partial \phi^{i} \partial \phi^{j}} K^{i j} d t
$$




$$
=G^{\mu^{\prime} \nu^{\prime}}\left\{-\frac{\partial L}{\partial \Phi^{\nu^{\prime}}} d t+E_{\nu^{\prime}, j} d Y^{j}\right\}+\frac{\partial E_{i}^{\mu^{\prime}}}{\partial \phi^{j}} K^{i j} d t
$$

while the constraint variables of course satisfy $d \Phi^{a}=0$. Here we assume ${ }^{[5]}$

$$
\nabla_{\mu^{\prime}} E_{i}^{\mu^{\prime}} \equiv \frac{1}{\sqrt{D}} \frac{\partial}{\partial \Phi^{\mu^{\prime}}}\left(\sqrt{D} E_{i}^{\mu^{\prime}}\right)=0
$$

where we use $\operatorname{det}\left(G_{\mu^{\prime} \nu^{\prime}}\right)=D$ and $\nabla_{\mu^{\prime}}$ is the covariant derivative in the manifold spanned by the independent variables, which assumption naturally leads metric condition $\nabla_{\mu^{\prime}} G^{\mu^{\prime} \nu^{\prime}}=0$. The Langevin equation (4.9) with Eqs. (4.8) and (4.10) is thereby reduced to

$$
d \Phi^{\mu^{\prime}}=G^{\mu^{\prime} \nu^{\prime}}\left\{-\frac{\partial L}{\partial \Phi^{\nu^{\prime}}} d t+E_{\nu^{\prime}, j} d Y^{j}\right\}+\frac{1}{\sqrt{D}} \frac{\partial}{\partial \Phi^{\nu^{\prime}}}\left(\sqrt{D} G^{\mu^{\prime} \nu^{\prime}}\right) d t
$$

which is GCT covariant in the manifold.

The Fokker-Planck equation made up of the independent variables is

$$
\frac{\partial P}{\partial t}=\left[\frac{\partial}{\partial \Phi^{\mu^{\prime}}} G^{\mu^{\prime} \nu^{\prime}}\left\{\frac{\partial \mathrm{L}}{\partial \Phi^{\nu^{\prime}}}-\frac{1}{\sqrt{D}} \frac{\partial \sqrt{D}}{\partial \Phi^{\nu^{\prime}}}+\frac{\partial}{\partial \Phi^{\nu^{\prime}}}\right\}\right] P
$$

which is no more than the rewriting of Eq. (3.14) and has only the same solution. Calculating the expectation value only of physical quantities, we can however change the Fokker-Planck equation (4.12) into the desirable form which has a finite solution. Physical quantity must not depend on the value of the multipliers $\lambda_{a}$, that is, the Poisson bracket of FCC and the physical quantity $\tilde{f}$ should be zero;

$$
\left\{\tilde{f}, F^{a}\right\}_{P . B .}=\frac{\partial \tilde{f}}{\partial \Phi^{b+N}}=0,
$$

which yields

$$
\langle d \tilde{f}\rangle=\left\langle\frac{\partial \tilde{f}}{\partial \Phi^{A}} d \Phi^{A}+\frac{\partial \tilde{f}}{\partial \Phi^{A+N}} d \Phi^{A+N}+\frac{\partial^{2} \tilde{f}}{\partial \Phi^{A} \Phi^{B}} g^{A B}+\frac{\partial^{2} \tilde{f}}{\partial \Phi^{A+N} \Phi^{B+N}} g_{A B}\right\rangle .
$$

The Fokker-Planck equation (4.12) hence loses the gauge variables $\Phi^{a+N}$ and is reduced to

$$
\frac{\partial \tilde{P}}{\partial t}=\left[\frac{\partial}{\partial \Phi^{A}} g^{A B}\left\{\frac{\partial L}{\partial \Phi^{B}}-\frac{1}{\sqrt{D}} \frac{\partial \sqrt{D}}{\partial \Phi^{B}}+\frac{\partial}{\partial \Phi^{B}}\right\}\right.
$$




$$
\left.+\frac{\partial}{\partial \Phi^{A+N}} g_{A B}\left\{\frac{\partial L}{\partial \Phi^{B+N}}-\frac{1}{\sqrt{D}} \frac{\partial \sqrt{D}}{\partial \Phi^{B+N}}+\frac{\partial}{\partial \Phi^{B+N}}\right\}\right] \tilde{P}
$$

Finding the finite solution identical with corresponding path integral distribution, we decompose the vielbein fields as

$$
\left\{\begin{aligned}
E_{i}^{\mu^{\prime}} & \equiv e_{\tilde{\mu}}^{\mu^{\prime} \epsilon_{i}^{\tilde{\mu}},} \\
E_{\mu^{\prime}, i} & \equiv e_{\mu^{\prime}}^{\tilde{\mu}} \epsilon_{\tilde{\mu}, i},
\end{aligned} \quad(\tilde{\mu}=M+1, \cdots, 2 N)\right.
$$

with

$$
\begin{gathered}
\epsilon_{i}^{\tilde{\mu}} \epsilon_{i}^{\tilde{\nu}}=\delta^{\tilde{\mu} \tilde{\nu}}, \\
\epsilon_{i}^{\tilde{\mu}} \epsilon_{\tilde{\nu}, i}=\delta_{\tilde{\nu}}^{\tilde{\mu}}, \\
\epsilon_{\tilde{\mu}, i} \epsilon_{\tilde{\nu}, i}=\delta_{\tilde{\mu} \tilde{\nu}} \\
e_{\tilde{a}+N}^{A}=e_{\tilde{a}+N}^{A+N}=e_{\tilde{A}}^{a+N}=e_{\tilde{A}+N}^{a+N}=0 .
\end{gathered}
$$

We then define new variables $C^{\tilde{a}+N}(\tilde{a}=1, \cdots, M)$ as

$$
\frac{\partial C^{\tilde{a}+N}}{\partial \Phi^{a+N}} \equiv e_{a+N}^{\tilde{a}+N}
$$

with which we can write the equilibrium solution of the Fokker-Planck equation (4.14), setting $C^{\tilde{a}+N}=0$, as

$$
\tilde{P}=\int D \lambda D \tilde{\lambda} \sqrt{D} \exp \left(-L+\lambda_{a} F^{a}+\tilde{\lambda}_{\tilde{a}+N} C^{\tilde{a}+N}\right)
$$

Taking notice that

$$
\operatorname{det}\left\{C^{\tilde{a}+N}, F^{a}\right\}_{P . B .}=\operatorname{det}\left(e_{a+N}^{\tilde{a}+N}\right)=\sqrt{D},
$$

the Fokker-Planck distribution (4.17) is identical with the path integral distribution 
which we obtain by imposing the gauge fixing conditions

$$
C^{\tilde{a}+N}=0, \quad(\tilde{a}=1, \cdots, M)
$$

upon the FCC

$$
F^{a}=0
$$

We thereby insist that our conjecture in $\S 3$ has been confirmed.

\section{6. $\S 5$. CONCLUSION}

In this paper we have formulated the SQM-ps on the classical basis of the canonical formalism; the Langevin equations in the phase space reduce to Hamilton equations in the classical limit. Furthermore our prescription of introducing constraints into the Langevin equations is consistent with Dirac's formalism. The Langevin equations with FCC, however, do not need to contain ordinary gauge fixing conditions, in place of which stochastic consistency conditions have been imposed and enabled to determine the Lagrange multipliers for the FCC. It has suggested that the stochastic consistency conditions should correspond to some gauge fixing. More information has been brought through the equilibrium FokkerPlanck distribution: After imposing the stochastic consistency conditions upon the FCC, we can obtain the finite values of physical quantities though the other quantities may diverge in the limit $t \rightarrow \infty$. We therefore conclude that imposing stochastic consistency conditions on FCC, being a little differnt from ordinary gauge fixing procedure, makes the FCC change into second class constraints.

\section{ACKNOWLEDGEMENTS}

I would like to thank K. Ikegami for stimulating discussions. 


\section{APPENDIX}

Here we show that our formulation is applicable to the systems with second class constraints. Following our prescription with gauge-fixed Hamiltonian

$$
\begin{aligned}
\tilde{H}_{G} & =H-\lambda_{a} F^{a}-\tilde{\lambda}_{\tilde{a}+N} C^{\tilde{a}+N} \\
& \equiv H-\bar{\lambda}_{\mathrm{a}} \bar{F}^{\mathrm{a}} \\
& (\mathrm{a}=1, \cdots, 2 M)
\end{aligned}
$$

we can straightforwardly reach the Langevin equation without the multipliers:

$$
d \phi^{i}=\bar{K}^{i j}\left\{-\frac{\partial L}{\partial \phi^{j}} d t+d Y^{j}\right\}-\frac{\partial \bar{F}^{\mathrm{a}}}{\partial \phi^{i}} \bar{D}_{\mathrm{ab}}^{-1} \frac{\partial^{2} \bar{F}^{\mathrm{b}}}{\partial \phi^{j} \partial \phi^{k}} \bar{K}^{j k} d t
$$

where

$$
\begin{gathered}
\bar{D}^{\mathrm{ab}} \equiv \frac{\partial \bar{F}^{\mathrm{a}}}{\partial \phi^{i}} \frac{\partial \bar{F}^{\mathrm{b}}}{\partial \phi^{i}}, \\
\bar{K}^{i j} \equiv \delta^{i j}-\frac{\partial \bar{F}^{\mathrm{a}}}{\partial \phi^{i}} \bar{D}_{\mathrm{ab}}^{-1} \frac{\partial \bar{F}^{\mathrm{b}}}{\partial \phi^{j}} .
\end{gathered}
$$

We can easily set up the Fokker-Planck equation, too:

$$
\frac{\partial \bar{P}(\phi, t)}{\partial t}=\frac{\partial}{\partial \phi^{i}} \bar{K}^{i j}\left\{\frac{\partial L}{\partial \phi^{j}}-\frac{\partial^{2} \bar{F}^{\mathrm{a}}}{\partial \phi^{j} \partial \phi^{k}} \bar{D}_{\mathrm{ab}}^{-1} \frac{\partial \bar{F}^{\mathrm{b}}}{\partial \phi^{k}}+\frac{\partial}{\partial \phi^{j}}\right\} \bar{P}(\phi, t),
$$

which has an equilibrium solution

$$
\bar{P}=\int D \bar{\lambda} \sqrt{\bar{D}} \exp \left(-L+\bar{\lambda}_{\mathrm{a}} \bar{F}^{\mathrm{a}}\right)
$$

The solution (A.1) should be identical with corresponding path integral distribution, which we can confirm with the following equations:

$$
\operatorname{det}\left\{C^{\tilde{a}+N}, F^{b}\right\}_{P . B .}=\operatorname{det}\left(\frac{\partial C^{\tilde{a}+N}}{\partial \Phi^{b+N}}\right)
$$




$$
\begin{aligned}
& =\operatorname{det}\left(\begin{array}{c}
\tilde{a}+N \\
e_{b+N}
\end{array}\right), \\
& \operatorname{det} \bar{D}^{\mathrm{ab}}=\operatorname{det} D^{a b} \cdot \operatorname{det}\left(\epsilon_{i}^{\tilde{a}+N} \epsilon_{i}^{\tilde{b}+N}\right) \\
& =\operatorname{det}\left(e_{b+N}^{\tilde{a}+N}\right)^{2} \text {. }
\end{aligned}
$$




\section{REFERENCES}

1. G.Parisi and Y.Wu, Sci.Sin. 24 (1981) 483

2. For a review, P.H.Damgaard and H.Hüffel, Phys.Rep. 152 (1987) 227;

M.Namiki, Stochastic Quantization (Springer-Verlag, 1992)

3. M.Namiki, I.Ohba, K.Okano and Y.Yamanaka, Prog. Theor. Phys. 69 (1983) 1580 ;

A.Nakamura, Prog. Theor. Phys. 86 (1991) 925

4. M.Namiki, I.Ohba and K.Okano, Prog. Theor. Phys. 72 (1984) 350

5. K.Ikegami, T.Kimura and R.Mochizuki, Nucl. Phys. B395 (1993) 371

6. S.Ryang, T.Saito and K.Shigemoto, Prog. Theor. Phys. 73 (1985) 1295;

A.M.Horowitz, Phys. Lett. 156B (1985) 89

7. I.Ohba, Prog. Theor. Phys. 77 (1987) 1267

8. K.Ito, Proc. Imp. Acad. 20 (1944) 519

9. R.Graham, Phys. Lett. 109A (1985) 209;

H.Rumpf, Phys. Rev. D33 (1986) 942

10. T.D.Lee and C.N.Yang, Phys. Rev. 128 (1962) 885

11. R.Mochizuki, Prog. Theor. Phys. 85 (1991) 407

12. R.Mochizuki, Prog. Theor. Phys. 88 (1992) 1233

13. P.A.M.Dirac, Lectures on Quantum Mechanics (Yeshiva Univ.,1964);

L.D.Faddeev, Theor. Math. Phys. 1 (1970) 1 